\title{
Exploring the Effects of Sexual Desire Discrepancy Among Married Couples
}

\author{
Brian J. Willoughby \\ Brigham Young University - Provo \\ Adam M. Farero \\ Brigham Young University - Provo \\ Dean M. Busby \\ Brigham Young University - Provo, dean_busby@byu.edu
}

Follow this and additional works at: https://scholarsarchive.byu.edu/facpub

Part of the Other Social and Behavioral Sciences Commons

\section{Original Publication Citation}

Willoughby, B. J., Farero, A., \& Busby, D. M. (2014). Exploring the effect of sexual desire discrepancy among married couples. Archives of Sexual Behavior, 43, 551-562.

\section{BYU ScholarsArchive Citation}

Willoughby, Brian J.; Farero, Adam M.; and Busby, Dean M., "Exploring the Effects of Sexual Desire Discrepancy Among Married Couples" (2013). Faculty Publications. 4621.

https://scholarsarchive.byu.edu/facpub/4621 


\title{
Exploring the Effects of Sexual Desire Discrepancy Among Married Couples
}

\author{
Brian J. Willoughby • Adam M. Farero • Dean M. Busby
}

Received: 18 June 2012 / Revised: 20 February 2013 / Accepted: 16 April 2013 / Published online: 18 September 2013

(C) Springer Science+Business Media New York 2013

\begin{abstract}
Previous studies have found associations between the individual discrepancy of desired sexual frequency and actual sexual frequency and relational outcomes among premarital couples. The present study extended this research by using a sample of 1,054 married couples to explore how actor and partner individual sexual desire discrepancy (SDD) scores were associated with relationship satisfaction, stability, communication, and conflict during marriage. All participants took an online survey which assessed both couple sexual dynamics and relationship outcomes. Findings suggested that higher actor individual SDD was generally associated with negative relational outcomes, including lower reported relationship satisfaction, stability, and more reported couple conflict. These effects were found after controlling for background factors, baseline sexual frequency and desire, and couple desire discrepancies. Some partner effects were also found and were generally in the same direction. Marital length did not moderate the effects found although gender moderated associations between individual SDD and reported couple communication. Negative associations between individual SDD and communication were particularly strong when the husband reported high discrepancies between desired and actual sexual frequency. Results suggested that higher individual sexual desire discrepancies among married individuals may undermine relationship well-being. Applications of these findings to a clinical setting are also discussed.
\end{abstract}

Keywords Sexual desire - Sexual satisfaction . Relationships $\cdot$ Sexual frequency $\cdot$ Marriage

B. J. Willoughby $(\bowtie) \cdot$ A. M. Farero · D. M. Busby

School of Family Life, Brigham Young University, 2081 JFSB,

Provo, UT 84602, USA

e-mail: brian.willoughby@byu.edu

\section{Introduction}

The connection between sexuality and relationship outcomes has been an area of frequent study among scholars. Multiple studies have suggested that sexual satisfaction and the wellbeing of relationships in general are positively correlated (Byers, 2005; Christopher \& Sprecher, 2000; Davies, Katz, \& Johnson, 1999). Although there are many elements of sexuality that are likely linked to relational outcomes, past research has suggested that higher frequency of sex (McNulty \& Fisher, 2008; Yucel \& Gassanov, 2010), a greater degree of individual sexual desire (Basson, 2002; Brezsnyak \&Whisman, 2004; Sprecher, 2002), and higher sexual satisfaction overall (Liu, 2003) are all associated with positive couple outcomes such as higher relationship satisfaction, commitment, and stability.

Several previous studies have also found that a difference between partners in their desired frequency of sexual behavior influences relational outcomes (Bridges \& Horne, 2007; Mark, 2012; Mark \& Murray, 2012). These studies have generally shown that higher desire discrepancies between couples are associated with negative relational outcomes. For example, Mark and Murray (2012) found that higher desire discrepancies between partners were related to lower male sexual satisfaction and lower female relationship satisfaction among a sample of college couples. Men's perception of a higher desire discrepancy between partners has also been related to lower relationship satisfaction (Davies et al., 1999). While these couplelevel discrepancies in desire are important, scholars have also argued that the combination of both individual sexual desire and reported sexual frequency may be uniquely influential to pathways through relationships and relational decision making (Willoughby \& Vitas, 2012).

Willoughby and Vitas (2012) utilized the term individual sexual desire discrepancy (SDD) to describe the difference between an individual's desire for sexual frequency and the 
actual sexual frequency in their relationship. In past research, a high individual SDD score has been used to capture an individual who desires a higher frequency of sexual activity than they are currently engaging in. Examining a sample of premarital dating couples, Willoughby and Vitas found that high individual SDD was associated with greater relationship satisfaction, but less stability. Follow-up analyses found that this unique finding was heavily moderated by relationship length and gender. First, the positive effect of individual SDD on satisfaction existing primarily when reported by females and, second, the effect of individual SDD on stability tended to diminishing over time for both genders, eventually becoming negative.

While this study was an important step forward in understanding how the combination of sexual desire and frequency may influence couple outcomes among premarital couples, no study to date has investigated if similar patterns might be found among couples after the marital transition. It is possible, for example, that within a marital framework where sexual intimacy may be more expected, unmet sexual desire may lead to more negative relational functioning and well-being. In the present study, we sought to explore how individual SDD influenced marital outcomes, including measures of satisfaction, stability, communication and conflict, among a national sample of married couples to understand how individual desire and frequency discrepancies may uniquely influence long-term marriages. In addition to these individual associations, we utilized data from both marital partners to examine an actor-partner model, allowing us to explore how both actor and partner reports of individual SDD influenced individual reports of relational outcomes. Finally, we explored how both gender and marital length moderated associations between individual SDD and relational outcomes.

\section{Marital Sexuality Versus Dating Sexuality}

Some sexuality research has provided evidence that sexual patterns change in marital relationships and that sexual behavior may have differing associations with outcomes among married couples. For example, married couples generally experience greater sexual satisfaction than their cohabiting and dating counterparts (Brown \& Booth, 1996; Christopher \& Sprecher, 2000; Waite, 1995). Although Smith et al. (2011) found that dating and married couples were not significantly different in terms of their desired frequency of sex, other scholars have reported that married couples were both more physically and emotionally satisfied with sex than their dating counterparts (Waite \& Joyner, 2001). Sexual frequency patterns also tend to shift after marriage, with past research suggesting that married couples have sex less frequently than dating and cohabitating couples (Call, Sprecher, \& Schwartz, 1995; Waite, 1995; Yabiku \& Gager, 2009), although this may have less to do with relationship status and be more connected to the nat- ural decrease in sexual frequency across time as relationships progress. Those in dating relationships may report elevated sexual frequency due to the excitement and novelty of a new sexual partnership, a novelty that would be expected to diminish in long-term relationships such as marriage.

In addition to central tendency differences in sexual satisfaction and frequency, some sexual behavior patterns may have differing impacts on relational outcomes based on marital status. For example, the frequency of physical intimacy may have less impact on marital relationships. Lower sexual frequency has been associated with higher rates of union dissolution in cohabiting couples compared to married couples (Yabiku \& Gager, 2009). Likewise, McNulty and Fisher (2008) found that sexual frequency was not significantly related to satisfaction among married couples. In fact, despite having a lower frequency of sex than both dating and cohabitating couples, married couples have higher physical sexual satisfaction with their partner than dating couples (Waite, 1995). Taken together, although increased sexual frequency is generally associated with positive relationship outcomes, some empirical evidence suggests that married couples appear to report greater physical and emotional satisfaction compared to unmarried couples despite a generally lower sexual frequency. This may suggest that the stability and well-being of the marital union may be less strongly based upon sexual frequency alone and more tied to other elements of the couple's relationship such as emotional intimacy or engagement in non-coital sexual behavior.

Despite these differences, not all aspects of sexuality appear to behave differently in married and unmarried populations. Unlike sexual frequency, the relationship between sexual desire and relational outcomes appears to be similar for both marital and dating couples. Among both types of couples, previous research has continuously shown a strong positive association between higher sexual desire and better relational outcomes (Brezsnyak \& Whisman, 2004; Byers, 2005; Davies et al., 1999; Gatzeva \& Paik, 2011; McNulty \& Fisher, 2008; Smith et al., 2011; Sprecher, 2002; Yeh, Lorenz, Wickrama, Conger, \& Elder, 2006). Sexual satisfaction has also been found to be positively associated with sexual desire in both dating and married populations (Davies et al., 1999; Smith et al., 2011). Specifically, the greater the difference between a dating couple's individual sexual desire, the greater the overall relationship and sexual dissatisfaction of the couple (Davies et al., 1999).

\section{Current Limitations and Present Study}

The present study contributes to the scholarly literature by addressing several current limitations in this area of scholarship. We take the concept of individual SDD and apply it to a previously unexplored sample-married couples. While previous studies have shown that actual (Mark, 2012; Mark \& Murray, 2012) or perceived (Davies et al., 1999) couple level 
discrepancies are generally harmful to couple relationships, only two previous studies (Santtila et al., 2008; Willoughby \& Vitas, 2012) have explored the effect of individual sexual desire discrepancies on couple outcomes and no previous study has focused exclusively on married couples.

We also expand the scope of measurement compared to previous studies by considering not only generalized assessments of relational outcomes (relationship satisfaction and stability) but two relational assessments (negative conflict and positive communication) that tap more day-to-day couple dynamics. While individual SDD has been previously associated with general assessments of relationship quality (Willoughby \& Vitas, 2012), it is unknown if such an association will carry over into more dynamic assessments of couple process. Like Willoughby and Vitas, in the present study, we utilized the Actor-Partner Interdependence Model (APIM) (Cook \& Kenney, 2005; Kenney \& Cook, 1999) to explore both actor and partner effects within a couple dataset. The APIM suggests that within an interdependent couple system, each partner's behaviors, values, and attitudes will have an effect on not only their own behavior and attitudes but also their partner's behavior and attitudes. Applied to the present study, the APIM would suggest that while an individual's SDD would influence their own perception of their relationship (actor effect), their partner's individual SDD would have its own unique effect on their perception of the relationship (partner effect). This conceptual model is illustrated in Fig. 1.

Previous scholarship on actor and partner SDD effects allowed us to generate specific hypotheses regarding the effect of individual SDD among married couples. For example, higher actor and partner SDD has been associated with negative couple outcomes among couples who had been dating for long periods of time (Willoughby \& Vitas, 2012). While high individual SDD may motivate individuals in early dating relationship to put more resources into their relationship, as that relationship progresses, a large difference between one's desired and actual sexual frequency may lead to frustration, resentment and anger as individuals are forced to deal with their unmet

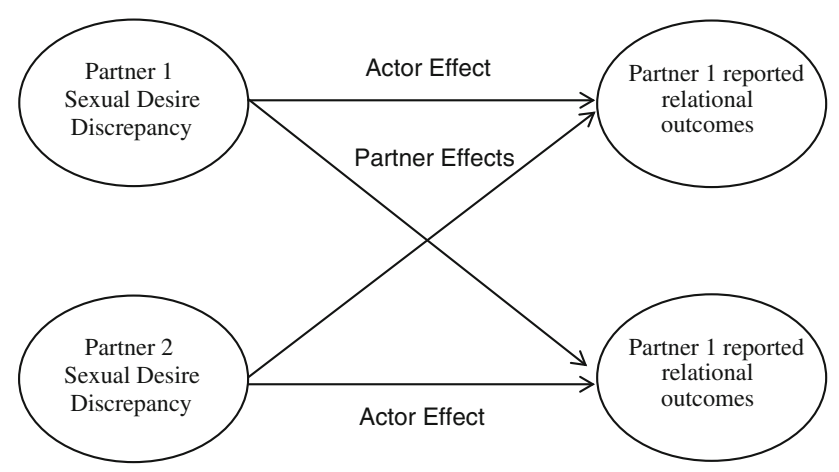

Fig. 1 Conceptual model of actor-partner effects between individual SDD and relational outcomes sexual expectations. Likewise, a marital partner's high individual SDD may cause them to engage in more hostile, negative relational behavior, lowering their partner's evaluation of the relationship. Thus, we propose the following two general hypotheses:

Hypothesis 1 Among married individuals, one's own higher desire for sexual frequency than actual sexual frequency (high actor SDD) will be negatively associated with one's own perception of relationship satisfaction, relationship stability, and positive communication and will be positively correlated with negative conflict.

Hypothesis 2 Among married individuals, a partner's higher desire for sexual frequency than actual sexual frequency (high partner SDD) will be negatively associated with one's own perception of relationship satisfaction, relationship stability, and positive communication and will be positively correlated with negative conflict.

Another contribution of the present manuscript is that while previous studies have found general associations between individual SDD and relational outcomes, no previous study has tested to see if the actual discrepancy between desired frequency and actual frequency is uniquely associated with relational outcomes after baseline assessments of sexual desire and sexual frequency are accounted for or after controlling for couple-level desire discrepancies. Previous associations between individual SDD and relational outcomes may have been predominantly driven by the general baseline effect of both sexual frequency and sexual desire and not uniquely by the discrepancy between these variables. Thus in the present study we not only tested if individual SDD was associated with relational outcomes among married couples, but also tested to see if such a discrepancy was still associated with such outcomes once the baseline effect of sexual desire and sexual frequency and couple-level desire discrepancies were accounted for. We propose the following additional hypothesis:

Hypothesis 3 Higher actor and partner SDD will be associated with one's own relational outcomes even after baseline sexual desire and sexual frequency scores and couple-level desire discrepancies are accounted for.

In addition to these three general hypotheses, in the present study we also explore the possible moderating effect of both gender and marital length. Previous sexuality research on married couples has long suggested important gender differences. For example, the general decline in sexual desire and sexual satisfaction seen among wives has long been assumed to be unique and different than desire and satisfaction trajectories of husbands (Elliott \& Umberson, 2008; Greeff \& Malherbe, 2001; Liu, 2003; Sims \& Meana, 2010). Qualitative data have also suggested that married women often view sex as an obligation instead of an activity of pleasure (Sims \& Meana, 2010). 
Specifically in relation to individual SDD, some research suggests that gender moderates the relationship between individual SDD and relational outcomes (Willoughby \& Vitas, 2012). In their sample of premarital dating couples, Willoughby and Vitas found that high female SDD was related to positive outcomes in early dating relationships but high male SDD scores were associated with negative outcomes in later dating relationships. Due to these specific findings, we suggest that male SDD may have a stronger association with relational outcomes among a sample of married couples who have likely been together for more than several years. We propose the following hypothesis:

Hypothesis 4 Male SDD scores will have a stronger negative association with marital outcomes compared to female SDD scores.

Additionally, sexual frequency generally declines with age and marital length (Call et al., 1995; Gager \& Yabiku, 2010). Middle-aged and older men expressed greater dissatisfaction with sexual frequency than their younger counterparts, again pointing to a decline in sex with relationship length (Smith et al., 2011). Related to individual SDD, Willoughby and Vitas (2012) found evidence of relational length moderation, finding an escalating negative association between individual SDD and relational outcomes as relationships progressed. These results suggested that as dating relationships progress and leave the initial dating and relationship formation stage, high individual SDD may be a liability in terms of couple outcomes. This effect may continue in marriages, as those in long-term marriages perceive higher individual SDD contributing to increased couple problems over time. However, as noted earlier, declining sexual frequency may not impact marital relationships as drastically as dating relationships (McNulty \& Fisher, 2008). Thus, it is unknown if marital length will operate in ways similar to dating length in regard to the effect of individual SDD or operate based on the general research on marital sexual frequency. For the present study, we assume that individual SDD among married couples will follow the patterns found by Willoughby and Vitas' (2012) study of individual SDD and propose the following final hypothesis:

Hypothesis 5 Marital length will moderate the relationship between individual SDD and relational outcomes with the negative effect of individual SDD becoming greater at longer marital lengths.

\section{Method}

\section{Participants}

Participants for this study included 2,108 married individuals who formed 1,054 unique mixed-sex couple pairs. These couples were sampled across the United States. These individuals formed couple pairs who took the RELATE (see below) instrument online (Busby, Holman, \& Taniguchi, 2001) between the years 2006 and 2011. The largest racial group was White (77.4\%) followed by Black (5.8\%), Asian (5.3\%), and Latino $(5.3 \%)$ participants. The largest religious denomination within the sample was Protestant (36.6\%). Twenty percent of the sample had been married for 2 years or less while $10 \%$ had been together for more than 20 years. Most couples (17.6\%) had been together between 3 and 10 years. About $20 \%$ of the sample reported a yearly personal income of less than $\$ 20,000$ while $14.8 \%$ of the sample reported a personal yearly income of more than $\$ 140,000$. The average age of the sample was 39.3 years $(S D=10.91)$ with a range from 19 to 76 . More detailed information on the sample is shown in Table 1.

Procedure

All participants completed an appropriate consent form prior to the completion of the RELATE instrument and all data collection procedures were approved by the institutional review board at the authors' university. Individuals completed RELATE online after being exposed to the instrument through a variety of settings. The RELATE assessment is a couple assessment designed to assess and provide feedback to those in romantic relationships. After taking the RELATE, couples are provided with feedback on their relationship strengths and weaknesses that they can utilize either on their own or in conjunction with a third party (i.e. religious leader, clinician). Some participants were referred to the online site by their instructor in a university class, others by a relationship educator or therapist, and some participants found the instrument by searching for it on the web. Participants were instructed to complete the assessment alone and to not discuss their responses with their partner. We refer the reader specifically to Busby et al.'s (2001) discussion of the RELATE for detailed information regarding the theory underlying the instrument and its psychometric properties.

\section{Measures}

\section{Demographics}

Sexuality within relationships varies by both race (Dariotis, Sifakis, Pleck, Astone, \& Sonenstein, 2011) and socioeconomic factors (Owen, Rhoades, Stanley, \& Fincham, 2010). Thus we control for both race and income in our analyses. Income was assessed by one item, which asked participants to indicate their current yearly gross income before taxes and deductions. Response ranged from 0 (none) to 9 ( $\$ 160,000$ and above). Participants were asked to identify the race with which they identified themselves. Responses were then dummy-coded for analysis with "white" being the reference group. Marital length was assessed by one item, which asked "How long have you and your partner been married?" Responses ranged from 1 
Table 1 Means and proportions for study sample (individual $n=2,108$ )

\begin{tabular}{|c|c|c|c|}
\hline Variable & $n(\%)$ & $M$ & $S D$ \\
\hline \multicolumn{4}{|c|}{ Individual sexual desire discrepancy ${ }^{\mathrm{a}}$} \\
\hline Male & & $1.13^{*}$ & 1.25 \\
\hline Female & & .526 & 1.12 \\
\hline \multicolumn{4}{|l|}{ Sexual engagement } \\
\hline Male & & $6.23 *$ & 2.15 \\
\hline Female & & 5.69 & 2.27 \\
\hline \multicolumn{4}{|c|}{ Couple desire discrepancy } \\
\hline Male & & 1.09 & 1.04 \\
\hline Female & & 1.08 & 1.03 \\
\hline \multicolumn{4}{|l|}{ Personal income ${ }^{\mathrm{b}}$} \\
\hline$<\$ 40,000$ & $769(36.5)$ & & \\
\hline$\$ 40,000-\$ 100,000$ & $797(37.8)$ & & \\
\hline$>\$ 100,000$ & $542(25.7)$ & & \\
\hline \multicolumn{4}{|l|}{ Relationship length } \\
\hline $0-6$ months & $177(8.3)$ & & \\
\hline $6-12$ months & $83(3.9)$ & & \\
\hline $1-5$ years & $365(17.1)$ & & \\
\hline $6-10$ years & $185(8.7)$ & & \\
\hline More than 10 years & $445(20.9)$ & & \\
\hline \multicolumn{4}{|l|}{ Race (male) } \\
\hline White & $827(77.2)$ & & \\
\hline African American & $64(6.0)$ & & \\
\hline Asian & $55(5.1)$ & & \\
\hline Latino & $59(5.5)$ & & \\
\hline Other & $66(6.2)$ & & \\
\hline \multicolumn{4}{|l|}{ Race (female) } \\
\hline White & $828(77.7)$ & & \\
\hline African American & $59(5.5)$ & & \\
\hline Asian & $58(5.4)$ & & \\
\hline Latino & $54(5.1)$ & & \\
\hline Other & $67(6.3)$ & & \\
\hline \multicolumn{4}{|c|}{ Desired sexual frequency ${ }^{c}$} \\
\hline Male & & 3.68 & 1.20 \\
\hline Female & & 3.11 & 1.24 \\
\hline \multicolumn{4}{|c|}{ Actual sexual frequency ${ }^{\mathrm{c}}$} \\
\hline Male & & 2.55 & 1.29 \\
\hline Female & & 2.58 & 1.30 \\
\hline \multicolumn{4}{|c|}{ Relationship satisfaction $^{\mathrm{d}}$} \\
\hline Male & & $3.43^{*}$ & .884 \\
\hline Female & & 3.32 & .983 \\
\hline \multicolumn{4}{|c|}{ Relationship stability ${ }^{\mathrm{d}}$} \\
\hline Male & & $3.99 *$ & .818 \\
\hline Female & & 3.90 & .861 \\
\hline \multicolumn{4}{|l|}{ Couple conflict $^{\mathrm{d}}$} \\
\hline Male & & 2.36 & .549 \\
\hline Female & & 2.36 & .595 \\
\hline
\end{tabular}

Table 1 continued

\begin{tabular}{lcr}
\hline Variable & $M(\%)$ & $S D$ \\
\hline $\begin{array}{l}\text { Positive communication }{ }^{\mathrm{d}} \\
\text { Male }\end{array}$ & $3.61^{*}$ & .623 \\
Female & 3.73 & .669 \\
\hline a Sexual desire discrepancy values ranged from 0 to 6 & \\
b Income values ranged from 0 (none) to 9 (\$160,000 or more per year), \\
percentages are for total sample \\
c Desired and actual sexual frequency scales range from 0 to 6 \\
d Relationship satisfaction, stability, couple conflict and communica- \\
tion scales range from 1 to 5 \\
* Significant gender differences at the $p<.01$ level
\end{tabular}

(0-3 months) to 9 (31 years or more). Gender was also measured and coded $($ male $=0$; female $=1)$.

\section{Individual Sexual Desire Discrepancy}

In line with previous work (Willoughby \& Vitas, 2012), assessments of both sexual desire and sexual frequency were utilized to construct a measure of individual SDD. Desired sexual frequency was measured by one item which asked participants, "How often do you desire to have sexual intercourse with your partner?" Actual sexual frequency was assessed by one item, which asked participants, "About how often do you currently have sex with your partner?" Both items were measured on a 7-point scale with responses ranging from 0 (never) to 6 (more than once a day). Individual SDD scores were computed by subtracting an individual's response on the actual frequency of sexual intercourse with their partner from their response on the desired sexual frequency with their partner item. Responses for this item ranged from -6 to 6 . Positive numbers indicated more desire for sexual activity than current involvement while negative numbers indicated less desire for sexual activity than current involvement. A measurement of 0 indicated that desired sexual frequency was matching actual sexual frequency. High correlations between partners on assessments of actual sexual frequency ( $r=.79, p<.001)$ suggested a high amount of congruence in partner reports on sexual behavior.

\section{Sexual Engagement}

While individual SDD was theorized to uniquely contribute to couple outcomes, one of the limitations of Willoughby and Vitas' (2012) study of dating couples was that no test was done to see if individual SDD predicted outcomes once baseline rates of sexual desire and frequency were accounted for. To address this limitation, a control variable was created that summed participants scores on the sexual frequency and desire measures 
to create an overall assessment of sexual engagement. While the original scores for both sexual frequency and desire could be used as control variables, because these variables were directly used to create the discrepancy scores this can create multicollinearity problems whereas a combined score still captures the overall influence but does not produce problematic results. This technique has been used in the past to ameliorate the common argument that discrepancy scores are problematic as they may primarily reflect the underlying relationship between the variables used to calculate the discrepancy score, hence it is important to control for baseline levels of the variables under consideration (Busby, Holman, \& Niehuis, 2009). Once computed, scores on sexual engagement ranged from 0 to $12(M=$ $6.60, S D=2.27)$.

\section{Couple Desire Discrepancy}

As some previous research has suggested that discrepancies between couples may be important in the prediction of relationship well-being (Mark \& Murray, 2012), we also assessed the difference between partners on their reported desired frequency of sexual intercourse. To create this variable we subtracted an individual's score on the sexual desire item (described above) from their partner's response. Due to inherent correlation between this assessment and our measure of individual SDD and to avoid issues related to multicollinearity, we took the absolute value of this difference. Scores on this scale ranged from 0 to $5(M=1.08, S D=1.04)$.

\section{Couple Outcomes}

Four measures of couple outcomes were used to assess individual satisfaction with the relationship, individual perception of the stability of the relationship, the amount of conflict in the relationship and positive couple communication. Relationship satisfaction was assessed with seven items asking participants how satisfied they were with various aspects of their relationship (for example, in their sexual relationships and with the overall relationship). Items were rated on a 5-point scale ( $1=$ very dissatisfied to $5=$ very satisfied). Cronbach's $\alpha$ was in the acceptable range $(\alpha=0.91)$. The RELATE satisfaction measures employed in this study have shown high test-retest reliability (between 0.76 and 0.78 ) and validity data have consistently shown that this scale is highly correlated with an existing relationship satisfaction and quality scale (Revised Dyadic Adjustment Scale) in both cross-sectional and longitudinal research (Busby et al., 2001, 2009).

Relationship stability was assessed by averaging three items, which asked participants how often the following three things had happened in their relationship: "How often have you thought your relationship (or marriage) might be in trouble?", "How often have you and your partner discussed ending your relationship (or marriage)?", and "How often have you broken up or separated and then gotten back together?"Responses ranged from 1 (never) to 5 (very often). These items were reverse coded so that higher scores indicated more stability. These items were adapted from earlier work by Booth, Johnson, and Edwards (1983). Cronbach's $\alpha$ was in the acceptable range $(\alpha=0.77)$. Previous studies have shown this scale to have test-retest reliability values between 0.78 and 0.86 , to be appropriately correlated with other relationship quality measures, and to be valid for use in cross-sectional and longitudinal research (Busby et al., 2001, 2007, 2009).

Couple conflict was assessed with twelve items asking participants how often they have conflict in specific areas such as financial matters, intimacy/sexuality, and time spent together, among others. The responses were measured on a 5-point scale ranging from 1 (never) to 5 (very often). Cronbach's $\alpha$ was in the acceptable range $(\alpha=0.78)$. Positive communication was assessed by asking participants eight items relating to their overall communication patterns. Sample items included: "I am able to listen to my partner in an understanding way," "When I talk to my partner I can say what I want in a clear manner," and "I sit down with my partner and just talk things over." Responses were measured on a 5 -point scale $(1=$ never to $5=$ very often $)$. In terms of test-retest and validity information on this scale, the communication items have been shown to have test-retest values between 0.70 and 0.83 and were appropriately correlated with a version of a commonly used Relationship Quality measure as predicted (Busby et al., 2001). Also this scale has been shown in longitudinal research to be predictive of couple outcomes and is amenable to change in couple intervention studies that focus on communication (Busby, Ivey, Harris, \& Ates, 2007). Cronbach's $\alpha$ was again in the acceptable range $(\alpha=0.87)$.

\section{Data Analysis}

Data analysis was undertaken in several steps. First, individual SDD scores were descriptively examined within the sample to gauge and compare the relative discrepancy between sexual desire and sexual frequency within a married sample. Next, in order to explore how individual SDD scores were related to couple outcomes and also explore cross-partner effects, actor and partner effects were explored utilizing Kenny's APIM (Kenny \& Cook, 1999). This model helps to both conceptualize and control for inherent couple dependency. In addition, the APIM helps model cross-partner effects. According to the APIM, individuals within a dyad will influence not only their own behavior and outcomes, but their partners as well. To explore this dynamic, data were arranged so that each individual was represented in the dataset twice, once as an actor and once as a partner.

Preliminary analyses suggested that data were not independent due to the presence of couple data. For example, individual scores within dyads were highly correlated (relationship 
satisfaction: $r=.71, p<.001$; relationship stability: $r=.76$, $p<.001)$ suggesting the need to control for dependency. We utilizing mixed regression techniques which allowed for the control of couple membership, utilizing gender as a withincouple repeating measure. Both actor and partner SDD scores were allowed in these regression models to predict individual outcome scores. Mixed regression models were set up to be hierarchical with three steps. The first model included only actor and partner SDD scores to explore basic associations between individual SDD and marital outcomes. Next, control variables were included to see if actor and partner effects remained once possible selection effects were accounted for. Control variables included race, marital length, gender, and income. Final models included all controls plus assessments of sexual engagement and couple level desire discrepancies to see if individual SDD was uniquely associated with couple outcomes once these factors were taken into account. At each step, model fit indices (AIC, BIC, -2 restricted log likelihood) were examined to see if model fit improved. In all models run, full models produced the best fitting models.

Since previous studies exploring individual SDD among dating couples (Willoughby \& Vitas, 2012) found that gender and relationship length may moderate relationships between individual SDD and couple outcomes, such interactions were also explored in the current study. When interactions existed, simple slope analyses (Aiken \& West, 1991) were utilized to explore moderation effects. For all interaction models, continuous variables were mean centered.

All variable and scales were tested for normality and all had adequate skewness and kurtosis values to suggest a normal distribution. One exception was found in regard to actor and partner SDD values which were slightly skewed due to the presence of outliers. Individuals who had SDD values \pm 3 SDs from the mean were removed. This removed 22 individuals ( 11 couples) or roughly $1 \%$ of the sample. Analyses suggested that these individuals did not differ from other individuals in the sample in terms of race, gender or income. Preliminarily analyses also suggested that the pattern of results presented was not altered regardless of if these data points were included. Data were assumed to be missing at random. Variables utilized in the present study had very few missing values. All variables utilized had less than $1 \%$ missing data. Preliminary analyses exploring missing data suggested that missing responses on independent variables were not related to differing values on relational outcomes, providing evidence for our assumption of missing at random responses. Based on these analyses, list-wise deletion was utilized in all analyses.

Due to the large sample size and to protect against the possibility of finding significant results that are not found in the true sampling population (Type 1 error), we have adapted a more conservative approach to $p$ value cut-offs. Instead of a typical cut-off of .05 , for the reported regression models we considered regression coefficients significant at the $p<.001$ level and marginally significant at the $p<.01$ level. We note where $p$ values were less than .05 but do not report them as significant.

\section{Results}

\section{Descriptive Results of Sexual Desire Discrepancy}

Before predictive models were run, descriptive statistics regarding individual SDD scores were explored. Husband SDD scores $(M=1.13 ; S D=1.25)$ were generally higher than wives SDD scores $(M=0.526 ; S D=1.12)$. This difference was significant, $t(2,101)=11.74, p<.001$. These averages are slightly lower than those found by Willoughby and Vitas (2012) among nonmarital couples suggesting that married individuals report less individual discrepancy between desired and actual sexual frequency. Thirty-seven percent of males in the sample reported an individual SDD score of zero indicating a desired sexual frequency that matched actual sexual frequency. Females indicated even more congruence between desired and actual sexual frequency levels with $52 \%$ of the wives having a zero on the individual SDD scale. While exploring couple level descriptive statistics, we found that males were more likely to report a higher individual SDD score than their spouses. Forty-eight percent of husbands reported a higher individual SDD score than their wives compared to just $18 \%$ of wives who reported a higher individual SDD than their husbands.

\section{Predicting Marital Outcomes}

Baseline models predicting relationship satisfaction found significant negative actor $(b=-0.19, t=-11.57, p<.001)$ and partner $(b=-0.16, t=-9.96, p<.001)$ associations between individual SDD scores and reported satisfaction. These results suggested that higher actor and partner individual SDD scores were associated with less reported relationship satisfaction. These actor $(b=-0.12, t=-5.62, p<.001)$ and partner $(b=$ $-0.06, t=-2.78, p=.041)$ effects remained even after demographic controls were added to the models. Table 2 summarizes final regression models which also included controls of sexual engagement and couple desire discrepancies. Actor effects remained significant, although our more conservative $p$-value suggested that partner effects were no longer significant.

For baseline assessments of relationship stability, negative associations were also found for both actor $(b=-0.12, t=$ $-7.75, p<.001)$ and partner $(b=-0.12, t=-8.07, p<.001)$ SDD scores. Higher actor and partner SDD scores were associated with less perceived relationship stability. These actor and partner effects remained significant after accounting for demographic controls and sexual engagement. Final model results are summarized in Table 2. Like relationship satisfaction results, once all controls were added to the final model, 
partner effects were only marginally significant with our more conservative $p$-value criteria.

Baseline models predicting couple conflict suggested positive associations between couple conflict and actor/partner SDD scores. Higher actor $(b=0.07, t=3.69, p<.001)$ and partner ( $b=0.07, t=4.17, p<.001)$ SDD scores were significantly associated with higher amounts of couple conflict. These effects again remained significant once demographic controls were accounted for. In the final model, once all controls were added to the model, both actor and partner effects were only marginally associated with reports of couple conflict with results again summarized in Table 2.

Finally, models predicting positive communication continued to show similar results. Higher actor $(b=-0.07, t=-5.95$, $p<.001)$ and partner $(b=-0.06, t=-4.99, p<.001)$ SDD scores were significantly associated with less positive communication. These effects remained after controlling for demographic controls. However, unlike previous relational outcomes, once sexual engagement and couple desire discrepancy were accounted for, actor and partner SDD was no longer significantly associated with positive communication suggesting that individual SDD did not provide meaningful associations with couple communication beyond baseline sexual frequency and desire and couple desire discrepancy.

In summary, even after controlling for demographic factors, sexual engagement and couple desire discrepancy, individual SDD was still significantly associated with most relational outcomes with the exception of couple communication. Higher actor SDD was associated with lower reported relationship satisfaction, lower stability, and more conflict providing support for Hypotheses 1 and 3. Similar partner effects were also found although they tended to be weaker than actor effects providing partial support for Hypothesis 2 . Associations between sexual engagement and couple desire discrepancy were generally in the expected direction. Higher reports of actor sexual engagement (reported sexual frequency and desired frequency) were associated with higher relationship satisfaction, stability, and communication. Higher discrepancies between partners on their reported desire for sexual frequency were associated with lower individual reports of relationship satisfaction, less relationship stability and less positive communication.

\section{Moderating Effect of Gender and Marital Length}

To test for gender moderation, a gender by actor/partner SDD interaction term was included into final regression models. Interactions with both actor and partner SDD terms were explored across all outcomes. Results are summarized in Table 3. Only one significant gender interaction was found for actor SDD for the model predicting couple communication suggesting weak evidence of Hypothesis 4. Post-hoc simple slope analyses were utilized to explore the relative effect of male and female actor
SDD scores on couple communication scores. Higher actor SDD did not have a significant association with positive communication for wives $(\beta=-0.01, t<1)$ but was associated with less positive communication among husbands $(\beta=-0.19$, $t=-6.06, p<.001)$. This interaction is shown in Fig. 2. To summarize, while higher actor SDD scores were not generally associated with couple communication in final models, these interaction effects suggested that such a relationship may be present but only for men.

Next, similar interaction effects were investigated between marital length and actor/partner SDD scores. Unlike interactions with gender, there was no evidence of marital length significantly moderating the effect between individual SDD and relational outcomes providing no evidence in favor of Hypothesis 5. None of the actor SDD by marital length interactions were significant. Similar to actor SDD interactions, no significant interaction was found between partner SDD scores and marital length. These results suggest that the effect of actor and partner SDD on marital outcomes are stable across differing marital lengths.

\section{Discussion}

Results of the present study provide important new information regarding the effect of individual SDD among marital couples. While previous studies of dating couples found evidence that high individual SDD may be beneficial to premarital relationships in the early stages of couple formation (Willoughby \& Vitas, 2012), results from the present study found no such positive association among married couples. In fact, results largely confirmed Hypotheses 1 and 2 and suggested that high individual SDD for either partner was generally associated with less satisfaction, less stability, less positive communication and more couple conflict among married couples with actor effects being the most robust. Distinctive to the present study, most SDD actor effects on relational outcomes were found to be unique, even when controlling for the baseline effect of both sexual frequency and sexual desire and couple level desire discrepancy providing additional support for Hypothesis 3 . Thus, although sexual frequency and desire (Brezsnyak and Whisman 2004; McNulty \& Fisher, 2008; Sprecher, 2002) and couple desire discrepancies (Mark, 2012; Mark \& Murray, 2012) are important indicators of couple well-being, results from the present study continue to suggest that individual SDD is an important and unique element of couple sexuality that is associated with the perception of one's relationship. Combined with the results of Willoughby and Vitas (2012), results suggested that high discrepancies between desired and actual sexual frequency may only be beneficial during a small window, early in the dating process.

Results from the present study also provide continued support for the assertion that marital sexuality may be different 
Table 2 Final unstandardized coefficients for mixed regression models predicting marital outcomes

\begin{tabular}{|c|c|c|c|c|c|c|c|c|}
\hline \multirow[t]{2}{*}{ Variable } & \multicolumn{2}{|c|}{ Relationship satisfaction } & \multicolumn{2}{|c|}{ Relationship stability } & \multicolumn{2}{|c|}{ Couple conflict } & \multicolumn{2}{|c|}{ Positive communication } \\
\hline & $b$ & SE & $b$ & SE & $b$ & SE & $b$ & SE \\
\hline Race $^{\mathrm{a}}$ & -0.024 & 0.053 & 0.044 & 0.047 & -0.045 & 0.048 & 0.009 & 0.044 \\
\hline Income & -0.004 & 0.007 & -0.005 & 0.006 & -0.003 & 0.006 & -0.004 & 0.006 \\
\hline Relationship length & -0.016 & 0.013 & -0.018 & 0.013 & $0.046^{* *}$ & 0.012 & $-0.012 *$ & 0.009 \\
\hline Gender $^{\mathrm{b}}$ & $-0.122 * *$ & 0.035 & $-0.114 * *$ & 0.029 & 0.002 & 0.033 & 0.081 & 0.032 \\
\hline Actor sexual engagement & $0.107 * *$ & 0.013 & $0.033^{*}$ & $0.012 *$ & $-0.028^{\dagger}$ & 0.013 & $0.068 * *$ & 0.011 \\
\hline Partner sexual engagement & $0.030^{\dagger}$ & 0.013 & -0.006 & 0.012 & -0.009 & 0.013 & 0.013 & 0.011 \\
\hline Couple desire discrepancy & $-0.164 * *$ & 0.031 & -0.145 & $0.032 * *$ & 0.050 & 0.028 & $-0.066^{*}$ & 0.021 \\
\hline Actor SDD & $-0.129 * *$ & 0.023 & $-0.120 * *$ & 0.023 & $0.059^{*}$ & 0.020 & -0.031 & 0.018 \\
\hline Partner SDD & $-0.047^{\dagger}$ & 0.023 & $-0.058^{*}$ & 0.023 & $0.050 *$ & 0.020 & -0.008 & 0.018 \\
\hline
\end{tabular}

$S D D$ sexual desire discrepancy

${ }^{\text {a }}$ Other $=0$; White $=1$

b Male $=0 ;$ female $=1$

$* * p<.001 ; * p<.01 ; ;^{\dagger} p<.05$

than sexuality among unmarried couples. Willoughby and Vitas (2012, p. 484) suggested that high sexual desire in the absence of sexual frequency among unmarried couples may serve as a "motivator to enhance and support the relationship as it progresses toward higher sexual frequency". However, once couples have transitioned to marriage, sexual frequency may be viewed as not only a common occurrence but, in some cases, a "right." Many married individuals, particularly women, view marital sexual intimacy as an obligation (Sims \& Meana, 2010) which may change the effect of high individual SDD. High individual SDD early in a dating relationship may cause some individuals to put more resources into the relationship to increase sexual frequency. This finding may also be due to the fact that in a new relationship, high desire to become intimate with a new romantic partner is impeded by barriers of either access (not living in the same home) or custom (feeling obligated to wait a certain number of dates before initiating sexual intercourse). Once married, these same individuals may simply view desire and frequency discrepancies as opportunities to become dissatisfied with the relationship.

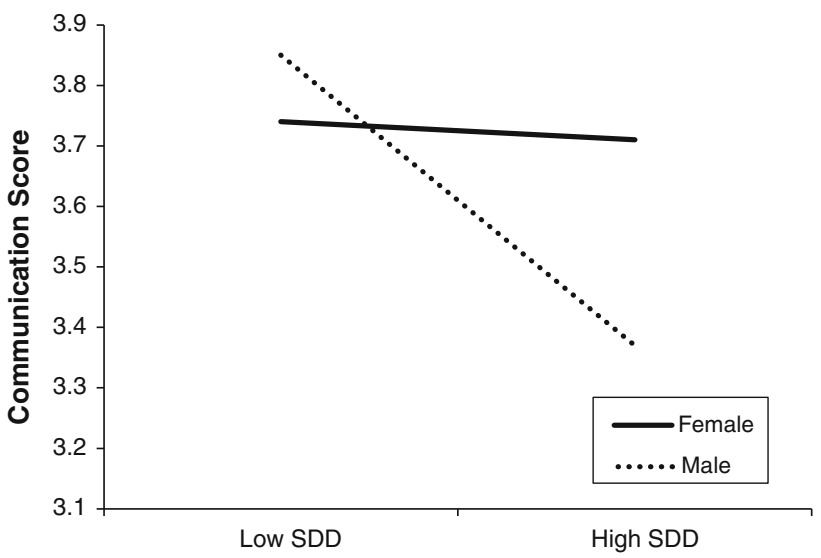

Fig. 2 Interaction between gender and individual actor SDD scores on positive communication. Data points based on \pm 1 SD on ISDD scale. Slope significant for only males $(p<.001)$

Like previous studies of individual SDD, some evidence of gender moderation was found between individual SDD and relational outcomes although this evidence in support of Hypothesis 4 was limited. Specifically, while high individual

Table 3 Gender and marital length interaction unstandardized coefficients for mixed regression models

\begin{tabular}{lccrr}
\hline Variable & Satisfaction & Stability & \multicolumn{2}{c}{$\begin{array}{l}\text { Conflict } \\
b\end{array}$} \\
& $b$ & $b$ & -0.009 & $b$ \\
ASDD*gender & 0.030 & -0.009 & 0.062 & $0.080^{*}$ \\
PSDD*gender & -0.053 & -0.044 & -0.013 & -0.007 \\
ASDD*length & 0.003 & -0.001 & -0.004 & 0.006 \\
PSDD*length & -0.006 & -0.002 & -0.001 \\
\hline
\end{tabular}

Note. Analyses controlled for race, sexual engagement, couple desire discrepancy gender, relationship length and income

$A S D D$ actor sexual desire discrepancy, $P S D D$ partner sexual desire discrepancy

$* p<.05 ; * * p<.01$ 
SDD was not generally associated with positive communication after controls were included into the models, moderation results suggested that such an effect may be present when husbands indicated a higher desire for sexual frequency compared to actual sexual frequency. This complements previous literature which has shown that husbands, but not wives, report lower sexual satisfaction as sexual frequency decreases over time (McNulty \& Fisher, 2008) and wives tend to report lower sexual desire than husbands (Basson, 2002; Baumeister, Catanese, \& Vohs, 2001). Given these findings and the fact that husbands in the present study were also found to be more likely to have higher individual SDD than their wives, results provide some limited support that gender differences exist in both how sexuality in marital relationships is perceived and how such sexuality impacts individual perceptions of the relationship based on gender. Indeed, interaction results with communication suggest that not only are husbands more likely to report larger discrepancies between desired and actual sexual frequency than their wives, but that such a difference was more likely to lead to negative marital communication compared to sexual desire discrepancies found among wives.

No support was found for Hypothesis 5 that marital length would moderate the relationship between individual SDD and marital outcomes, suggesting that the negative effect of individual SDD does not worsen or lessen over time. Willoughby and Vitas (2012) found that the effect of individual SDD did vary over time among dating couples, suggesting that individual SDD's influence on relational outcomes may eventually level during marriage or within other long-term relationships. The results of these two studies paint an interesting picture regarding the effect of individual SDD over the course of a relationship. Taken together, results suggest that in early relationships, high female SDD is generally associated with better relational outcomes. This effect tends to diminish and perhaps reverse within the first few years of dating where high male SDD may then have a negative effect on relational outcomes (see Willoughby \& Vitas, 2012). For couples that transition to marriage, high individual SDD continues to be associated with negative relational outcomes and in some cases high male SDD may be particularly linked to negative marital outcomes. Unlike dating relationships, within a marriage this negative effect appears to remain stable over time. It is also possible that this stabilization effect may be due to couples "selecting out" of marriage through divorce when high individual SDD has led to high relationship dissatisfaction and instability. When viewed from this alternative perspective, high individual SDD may not create worsening relational outcomes over time in a cross-sectional sample simply due to the fact that when such an effect occurs, those individuals experiencing a prolonged discrepancy between sexual frequency and desire may simply end the relationship. Longitudinal studies which can track the effect of individual SDD over time are needed to replicate and test cross-sectional patterns found in the present and previous studies.
Clinical Implications

The results of the current study also present some interesting clinical implications. The correlation of high individual SDD with negative marital outcomes highlights the influential nature of sex, and particularly individual SDD, on multiple aspects of a marriage. For this reason, it is important for clinicians to assess not only sexual frequency and sexual desire, but also individual SDD. Although not all instances of individual SDD will lead to negative marital outcomes, with open discussion of the topic individual SDD may be revealed as a source of conflict to be addressed within a clinical setting. Clients seeking marital counseling may be surprised to learn that marital issues seemingly unrelated to sex may actually stem from their spouse's unmet sexual expectations. This is logical, considering that if marital communication is impaired, one spouse may view sexual frequency as adequate, while being unaware of his or her spouse's dissatisfaction with the sexual relationship. Clinicians may be able to facilitate improved marital satisfaction by promoting discussion of underlying individual SDD and open communication among spouses about expectations for sexual frequency. Additionally, it could be important for clinicians to help clients address emotions and cognitions related to actor SDD, such as a sense of inadequacy or a view of a spouse as inconsiderate or selfish.

\section{Limitations and Future Directions}

Despite the important contributions of the present study, several limitations should be noted. First, sample limitations may have altered some of the associations found in the study. Although the sample included couples from across the United States, the sample utilized tended to be more educated and have more financial resources than would be found in a truly national representative sample. Additionally, the ethnic diversity of the sample was lacking with the present study being predominately white and not reflecting national racial proportions. Taken together, caution should be taken before generalizing the results of this study to couples from lower socio-economic environments and those from minority racial groups. Effect sizes were also generally small suggesting that individual SDD is only one component of relationship well-being. While results continue to suggest that individual SDD is an important, understudied aspect of relationship health, many other aspects of an individual's background and current relationships are interconnected to individual SDD scores and their effect.

Additionally, the cross sectional nature of the data does not allow for causal pathways to be established. While results incorporating marital length provide for crude estimates of how individual SDD may be differentially associated with relational outcomes across time, as previously noted alternative explanations for result patterns are also possible. Longitudinal studies that are able to track couples across time and across 
the marital transition are needed to understand how individual SDD influences relational outcomes across time. While results suggested the effect of individual SDD did not change significantly over time, longitudinal data would be needed to verify this claim and to provide a more dynamic understanding of individual SDD and its effect on relational outcomes. Daily diary studies tracking daily couple interactions and outcomes may be one way to tease out these patterns. It would also be helpful in future longitudinal studies to explore the possible reciprocal effects between individual SDD and relational outcomes. While lower individual SDD may in fact lower perceptions of relationship quality, it is also likely that lower evaluations of the relationship may decrease individual SDD by lowering individual sexual desire. This reciprocal effect should be a focus of future longitudinal scholarship.

\section{Conclusions}

Despite these limitations, the present study offers many significant contributions to the scholarly literature. Being the first study to examine the effect of individual SDD on marital outcomes, results from this study provide continued evidence that incongruence between desired frequency and actual sexual frequency appears to be a potential catalyst for negative couple outcomes and, within a marital context, possible future marital dissolution. Scholars should continue to seek to understand how discrepancies within and between partners might help us understand short-term and long-term relational trajectories and health.

\section{References}

Aiken, L. S., \& West, S. G. (1991). Multiple regression: Testing and interpreting interactions. Newbury Park, CA: Sage.

Basson, R. (2002). Women's sexual desire-Disordered or misunderstood? Journal of Sex and Marital Therapy, 28, 17-28.

Baumeister, R. F., Catanese, K. R., \& Vohs, K. D. (2001). Is there a gender difference in strength of sex drive? Theoretical views, conceptual differences, and a review of relevant evidence. Personality and Social Psychology Review, 5, 242-273.

Booth, A., Johnson, D. R., \& Edwards, J. N. (1983). Measuring marital instability. Journal of Marriage and the Family, 45, 387-394.

Brezsnyak, M., \& Whisman, M. A. (2004). Sexual desire and relationship functioning: The effects of marital satisfaction and power. Journal of Sex and Marital Therapy, 30, 199-217.

Bridges, S. K., \& Horne, S. G. (2007). Sexual satisfaction and desire discrepancy in same sex women's relationships. Journal of Sex and Marital Therapy, 33, 41-53.

Brown, S. L., \& Booth, A. (1996). Cohabitation versus marriage: A comparison of relationship quality. Journal of Marriage and Family, $58,668-678$.

Busby, D. M., Holman, T. B., \& Niehuis, S. (2009). The association between partner- and self enhancement and relationship quality outcomes. Journal of Marriage and Family, 71, 449-464.

Busby, D. M., Holman, T. B., \& Taniguchi, N. (2001). RELATE: Relationship evaluation of the individual, family, cultural, and couple contexts. Family Relations, 50, 308-316.
Busby, D. M., Ivey, D. C., Harris, S. M., \& Ates, C. (2007). Self-Directed, therapist-directed, and assessment-based interventions for premarital couples. Family Relations, 56, 279-290.

Byers, E. S. (2005). Relationship satisfaction and sexual satisfaction: A longitudinal study of individuals in long-term relationships. Journal of Sex Research, 42, 113-118.

Call, V., Sprecher, S., \& Schwartz, P. (1995). The incidence and frequency of marital sex in a national sample. Journal of Marriage and Family, 57, 639-652.

Christopher, F. S., \& Sprecher, S. (2000). Sexuality in marriage, dating and other relationships: A decade review. Journal of Marriage and the Family, 62, 999-1017.

Cook, W. L., \& Kenny, D. A. (2005). The actor-partner interdependence model: A model of bidirectional effects in developmental studies. International Journal of Behavioral Development, 29, 101-109.

Dariotis, J. K., Sifakis, F., Pleck, J. H., Astone, N. M., \& Sonenstein, F. L. (2011). Racial and ethnic disparities in sexual risk behaviors and STDs during young men's transition to adulthood. Perspectives on Sexual and Reproductive Health, 43, 51-59.

Davies, S., Katz, J., \& Jackson, J. L. (1999). Sexual desire discrepancies: Effects on sexual and relationship satisfaction in heterosexual dating couples. Archives of Sexual Behavior, 28, 553-567.

Elliott, S., \& Umberson, D. (2008). The performance of desire: Gender and sexual negotiation in long-term marriages. Journal of Marriage and Family, 70, 391-406.

Gager, C. T., \& Yabiku, S. T. (2010). The relationship between household labor time and sexual frequency. Journal of Family Issues, 31, 135163.

Gatzeva, M., \& Paik, A. (2011). Emotional and physical satisfaction in noncohabiting, cohabiting, and marital relationships: The importance of jealous conflict. Journal of Sex Research, 48, 29-42.

Greeff, A. P., \& Malherbe, H. L. (2001). Intimacy and marital satisfaction in spouses. Journal of Marital and Sex Therapy, 27, 247-257.

Kenny, D. A., \& Cook, W. (1999). Partner effects in relationships research: Conceptual issues, analytic difficulties and illustrations. Personal Relationships, 6, 433-448.

Liu, C. (2003). Does quality of marital sex decline with duration? Archives of Sexual Behavior, 32, 55-60.

Mark, K. P. (2012). The relative impact of individual sexual desire and couple desire discrepancy on satisfaction in heterosexual couples. Sexual \& Relationship Therapy, 27, 133-146.

Mark, K. P., \& Murray, S. H. (2012). Gender differences in desire discrepancy as a predictor of sexual and relationship satisfaction in a college sample of heterosexual romantic relationships. Journal of Sex and Marital Therapy, 38, 198-215.

McNulty, J. K., \& Fisher, T. D. (2008). Gender differences in response to sexual expectancies and changes in sexual frequency: A short-term longitudinal study of sexual satisfaction in newly married couples. Archives of Sexual Behavior, 37, 229-240.

Owen, J. J., Rhoades, G. K., Stanley, S. M., \& Fincham, F. (2010). "Hooking up" among college students: Demographic and psychosocial correlates. Archives of Sexual Behavior, 39, 653-663.

Santtila, P., Wager, I., Witting, K., Harlaar, N., Jern, P., Johansson, A., et al. (2008). Discrepancies between sexual desire and sexual activity: Gender differences and associations with relationship satisfaction. Journal of Sex and Marital Therapy, 34, 31-44.

Sims, K. E., \& Meana, M. (2010). Why did passion wane? A qualitative study of married women's attributions for declines in sexual desire. Journal of Sex and Marital Therapy, 36, 360-380.

Smith, A., Lyons, A., Ferris, J., Richters, J., Pitts, M., Shelley, J., et al. (2011). Sexual and relationship satisfaction among heterosexual men and women: The importance of desired frequency of sex. Journal of Sex and Marital Therapy, 37, 104-115.

Sprecher, S. (2002). Sexual satisfaction in premarital relationships: Associations with satisfaction, love, commitment, and stability. Journal of Sex Research, 39, 190-196. 
Waite, L. J. (1995). Does marriage matter? Demography, 32, 483-507.

Waite, L. J., \& Joyner, K. (2001). Emotional satisfaction and physical pleasure in sexual unions: Time horizon, sexual behavior, and sexual exclusivity. Journal of Marriage and Family, 63, 247-264.

Willoughby, B. J., \& Vitas, J. (2012). Sexual desire discrepancy: The effect of individual differences in desired and actual sexual frequency on dating couples. Archives of Sexual Behavior, 41, 477-486.

Yabiku, S. T., \& Gager, C. T. (2009). Sexual frequency and the stability of marital and cohabiting unions. Journal of Marriage and Family, 71, 983-1000.
Yeh, H., Lorenz, F. O., Wickrama, K. A. S., Conger, R. D., \& Elder, G. H. (2006). Relationships among sexual satisfaction, marital quality, and marital instability at midlife. Journal of Family Psychology, 20,339_ 343.

Yucel, D., \& Gassanov, M. A. (2010). Exploring actor and partner correlates of sexual satisfaction among marriage couples. Social Sciences Research, 39, 725-738. 